Received: 2020-08-25

Accepted: 2020-09-24

Published: 2021-03-31

\section{Ultrasound-Guided Alcohol Neurolysis For Treatment of Refractory Radial Neuralgia}

\author{
Ashwin V. Rangavajjula, MD' \\ Toyin Okanlawon, $\mathrm{MD}^{2}$ \\ Mercy Udoji, MD ${ }^{3}$
}

Background: The radial nerve is especially vulnerable to injury due to its location near the humerus. When acute pain becomes chronic and surgical repair is no longer an option, it is necessary to have non-opioid, nonpharmacological methods of pain relief.

Case Report: A 63-year-old man presented with severe radial nerve neuralgia secondary to traumatic left upper extremity injury 40 years ago. After several unsuccessful surgical interventions and failing medication management, he was sent to pain management as a last resort prior to elective amputation of the left upper limb. After initial evaluation, the patient underwent a diagnostic ultrasound-guided radial nerve block at the midhumeral level with complete but short-lasting relief of his pain. Shortly after, we performed ultrasoundguided alcohol neurolysis of the radial nerve at the same level. This treatment resulted in $100 \%$ pain relief for 10 months with gradual resumption of pain to previous levels at 12 months post neurolysis.

Conclusion: Alcohol neurolysis is an effective and viable option for the treatment of neuralgia resistant to other therapeutic modalities. To our knowledge, this is the first case report that describes the use of ultrasound to perform chemical neurolysis of the radial nerve.

Key words: Alcohol neurolysis, chronic pain, nerve ablation, neuralgia, neurolysis, opioids, radial nerve, traumatic injury, ultrasound

\section{BACKGROUND}

Radial nerve injuries are a challenge for surgeons and pain physicians alike. The proximity of the nerve to the humerus makes it especially susceptible to injury from humeral fractures. The radial nerve is also at risk during compressive and blast-type injuries (1). Radial neuralgia caused by physical trauma is usually transient, resolving over time with conservative therapy. In some cases, acute pain becomes chronic and is characterized by pain, paresthesia, and numbness along the distribution of the nerve.
Surgical management for an initial musculoskeletal injury is a first-line treatment option. Multiple surgeries that fail to correct the underlying deformity can cause more nerve inflammation and damage, thereby contributing to or creating refractory neuralgia. Unfortunately, when such patients can no longer benefit from surgery, they are referred to pain management clinics with little to no therapeutic options.

Most patients are successfully managed with a combination of neuropathic pain medications, opioids, muscle relaxants, or manual therapy. Over time, a subset of

From: ' Crystal Run Healthcare, Interventional Pain Management, Middletown, NY; ${ }^{2}$ MAK Anesthesia, Kennesaw, GA; ${ }^{3}$ Emory University School of Medicine/ Atlanta VA Medical Center, Department of Anesthesiology, Decatur, GA

Corresponding Author: Mercy Udoji, MD E-mail: mercy.udoji@va.gov

Disclaimer: There was no external funding in the preparation of this manuscript.

Conflict of interest: Each author certifies that he or she, or a member of his or her immediate family, has no commercial association (i.e., consultancies, stock ownership, equity interest, patent/licensing arrangements, etc.) that might pose a conflict of interest in connection with the submitted manuscript. 
patients with severe refractory pain may require surgical decompression or neurolysis. As we have become more aware of the adverse side effects and addictive risks of long-term opioid management, there is an obvious need for more opioid-sparing options. Other therapeutic options include steroid injections, pulsed radiofrequency lesions, or alcohol neurolysis, all with limited evidence supporting their use.

\section{CASE}

Consent to discuss the details of his case and care was provided by the patient.

A 63-year-old male veteran whose past medical history was notable for hypertension, diabetes mellitus type II, and Parkinson's disease was referred to our clinic with a chief complaint of severe left-handed pain in the distribution of the radial nerve. While he was in the military, he fell from an airplane wing in the 1970s and sustained severe left hand and arm injury that required multiple ( $>3$ ) surgeries. The pain in his left hand and forearm never resolved and continued to worsen since that time.

In 2008, he experienced traumatic amputation and reattachment of the left middle, ring, and index fingers, followed by hardware removal with external tenolysis of the fourth and fifth digits in the left upper extremity and neuroma excision in 2009. Seven years prior to his initial visit, he had a posterior interosseous neurectomy to reduce chronic pain, functional instability, and neuropathy with minimal improvement. Over time, he continued to experience severe pain, loss of grip strength, decreased temperature sensation, and persistent hypoesthesia and dysesthesia in the radial nerve distribution. He tried conservative therapies including physical therapy, immobilization, and pharmacotherapy with marginal benefit.

Five months prior to presentation, he underwent open neurolysis, excision of neuroma, and collagen conduit reconstruction and collagen wrapping of the left radial nerve for chronic intractable neuropathic pain. Unfortunately, his pain and functional disability worsened postoperatively to such an extent that he was no longer able to extend his digits and was unable to put on his clothes; his left hand was rendered "useless." At that time, his surgeon declined further intervention and referred him to our service with the understanding that elective amputation of the affected limb was his only remaining therapeutic option if his pain remained uncontrolled.
Upon presentation, his medication regimen consisted of pregabalin $250 \mathrm{mg} 3$ times a day, acetaminophen 500 mg 3 times a day as needed, and venlafaxine $150 \mathrm{mg}$ daily. He had tried duloxetine, diclofenac gel, lidocaine cream, opioids, and gabapentin in the past with little relief, or discontinued them due to side effects. Nonsteroidal agents were not an option for this veteran due to renal insufficiency.

His chief complaint was constant, twisting, sharp pain along the dorsal aspect of his left wrist and lateral aspect of his left hand, especially into digits 1, 2, and 3. The patient also described pain at the dorsal aspect of his left elbow that began after his last surgical procedure radiating to the same digits. His pain ranged from 8 to 10 on the Numeric Rating Scale (NRS-11) and any movement or pressure on the left hand below the elbow exacerbated the pain. In addition, he had difficulty extending his wrist and was unable to grip objects in his left hand. Previous, partially alleviating factors which included heat application and pain medications no longer worked. Per the patient, pain significantly interfered with his daily activities and worsened his mental health condition (depression).

Physical examination of his left hand revealed significant postoperative scarring and contracture of the left wrist and first 3 digits of the left hand. His left hand and forearms revealed multiple well-healed scars and a prominent dorsal skin incision. On exam he had severe tenderness to palpation at the radiocarpal joint and severe tenderness with light touch throughout the dorsal sensory branch of the radial nerve, lateral to his incision. On the ulnar aspect of his arm and forearm, the patient endorsed decreased sensation. He was unable to actively extend his digits due to pain but had good passive mobility of the metacarpal phalangeal joints on the left upper extremity. Complex regional pain syndrome was in the differential diagnosis but was ruled out due to lack of symptoms in any other nerves in the affected extremity.

As such, we proposed a diagnostic radial nerve block to confirm the etiology of pain and to guide future interventional options. After a thorough discussion of the risks and alternatives, which included motor weakness, increased pain, deafferentation pain, worsening dysesthesia, and hyperesthesia, the patient was agreeable to a diagnostic radial nerve block with planned progression to alcohol neurolysis if appropriate. Alcohol neurolysis was chosen in place of peripheral nerve stimulation because the technology was not available 
at our institution at the time this patient was evaluated. Because his symptoms extended to the elbow, we performed an ultrasound (US)-guided radial nerve block at the midhumeral level (Fig. 1). Following successful US-guided diagnostic and therapeutic block of the radial nerve with $5 \mathrm{~mL}$ of a solution containing $0.5 \%$ bupivacaine and $20 \mathrm{mg}$ methyl-prednisolone, the patient reported near complete relief of his pain lasting 3 weeks, with a return to his preintervention level of pain soon thereafter.

Due to his positive result, we made the decision to perform alcohol neurolysis 6 weeks after his initial block. The patient again underwent an US-guided radial nerve block at the midhumeral level. This time, once the nerve was identified, it was hydrodissected away from surrounding structures using $3 \mathrm{~mL}$ of a solution containing $2 \%$ lidocaine and sterile saline in a 1:1 ratio. After waiting 3 minutes, $5 \mathrm{~mL}$ of $100 \%$ alcohol was injected around the nerve.

At his one week follow-up, the patient endorsed numbness and lack of pain in the distribution of the left radial nerve, except for some residual pain at the base of his left thumb. His NRS-11 score at that visit was
2 of 10 . Within 3 months of his procedure, the pain at the base of his left thumb had completely resolved. The patient remained pain-free and was able to dress himself, use the affected limb to operate his electric wheelchair, open doors, etc. until 10 months after the injection, when pain began to recur.

\section{DISCUSSION}

The use of sonography to perform targeted injections for the relief of chronic pain is becoming more common (2). Alcohol neurolysis is a well-established treatment modality for the management of cancer-related pain (i.e., celiac plexus neurolysis). This technique is rarely used for other types of refractory, chronic pain syndromes. Even less common is the use of sonography to perform radiofrequency ablations or chemical (alcohol or phenol) neurolysis for painful conditions. There are cases in the literature reporting the use of US to perform radiofrequency ablation and alcohol neurolysis of painful stump neuromas $(3,4)$, meralgia paresthetica $(5,6)$, and for malignant abdominal wall pain (7). To our knowledge, this is the first case report to describe the use of US to perform chemical neurolysis of the radial

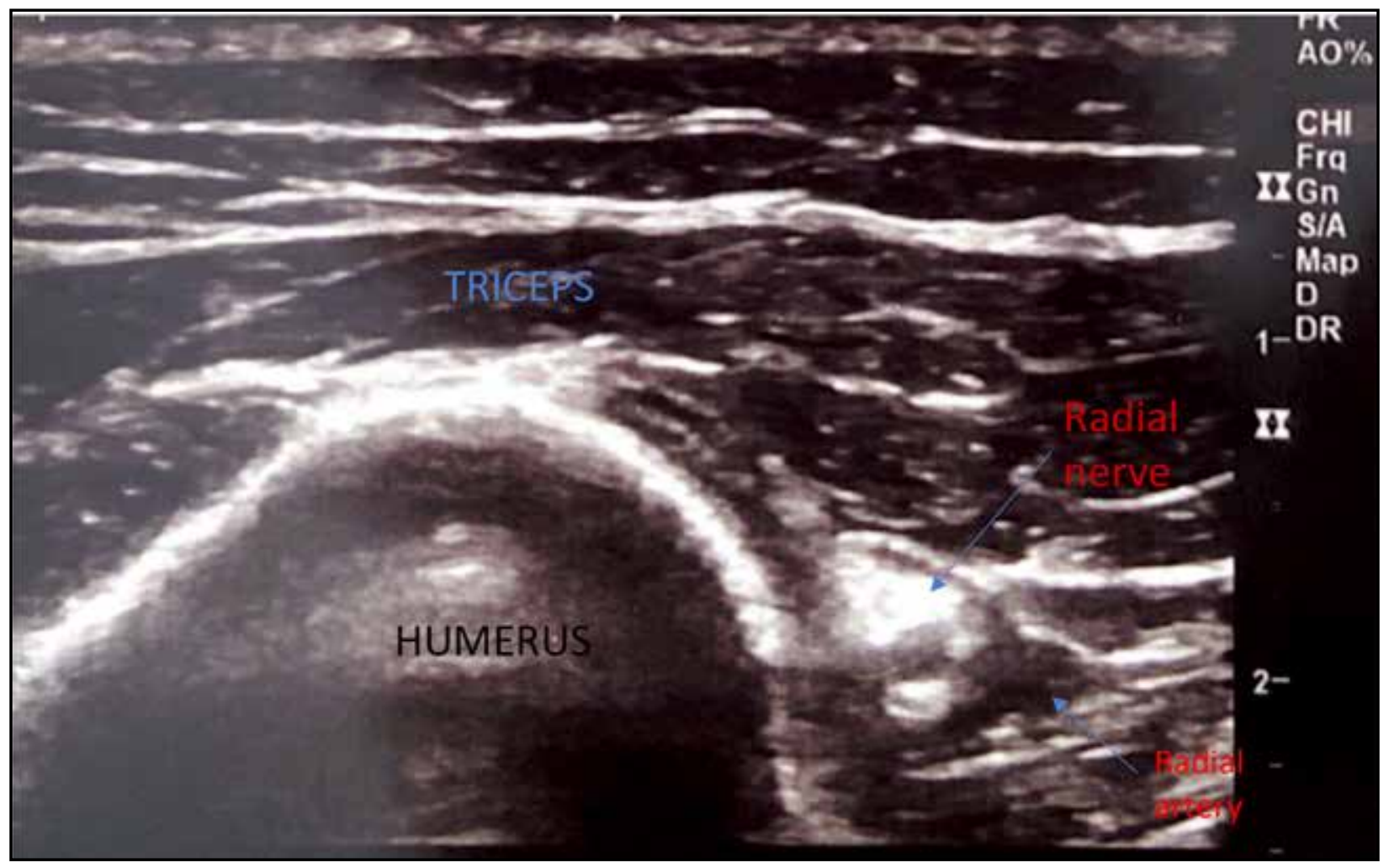

Fig. 1. Radial nerve and artery at midhumerus. Ultrasound image of the radial artery and nerve at the midhumeral level, therapeutic target for injections. 
nerve for long-term relief of intractable pain. Phenol was not considered for this patient due to limited availability at our institution.

The accidental blockade of other important structures or injury of vascular structures has been described during neurolysis. Our choice of US permitted real-time visualization and allowed us to avoid similar difficulties (8). Our patient did not experience any complications, but the risk of deafferentation pain, anesthesia dolorosa, or dysesthesia has been described in the literature (9).

\section{CONCLUSION}

US-guided neurolysis is a good option for patients with intractable, distal peripheral mononeuropathy who fail conservative therapy. This is a safe and effective procedure that provides long-lasting, opioid-free analgesia for affected patients.

\section{Authors' Contributions}

All of the authors participated in writing the manuscript and participated in the revision process and finalization of the manuscript

\section{REFERENCES}

1. Ljungquist $\mathrm{KL}$, Martineau $\mathrm{P}$, Allan $\mathrm{C}$. Radial nerve injuries. J Hand Surg Am 2015; 40:166-172.

2. Korbe S, Udoji EN, Ness TJ, et al. Ultrasound-guided interventional procedures for chronic pain management. Pain Manag 2015; 5:465-482.

3. Musson RE, Sawhney JS, Lamb L, Wilkinson A, Obaid H. Ultrasound guided alcohol ablation of Morton's neuroma. Foot Ankle Int 2012; 33:196-201.

4. Zhang Z, Xu Y, Xhou J, et al. Ultrasound-guided alcohol neurolysis and radiofrequency ablation of painful stump neuroma: Effective treatments for post-amputation pain. J Pain Res 2017; 10:295302.

5. Ahmed A, Arora D, Kochhar AK. Ultrasound-guided alcohol neurolysis of lateral femoral cutaneous nerve for intractable meralgia paresthetica: A case series. Br J Pain 2016; 10:232-237.

6. Chen CK, Phui VE, Saman MA. Alcohol neurolysis of lateral femoral cutaneous nerve for recurrent meralgia paresthetica. Agri $2012 ; 24: 42-44$

7. Hung JC, Azam N, Puttaniah V, et al. Neurolytic transversus abdominal plane block with alcohol for long-term malignancy related pain control. Pain Physician 2014; 17:E755-E760.

8. Konder H, Moysich F, Mattush W. An accidental motor blockade of the femoral nerve following a blockade of the lateral femoral cutaneous nerve. Reg Anaesth 1990; 13:122-123.

9. Kirazli $Y$, On AY, Kismali $B$, et al. Comparison of phenol block and botulinum toxin Type $A$ in the treatment of spastic foot after stroke: A randomized, double blind trial. Am J Phys Med Rehabil 1998; 77:510-515. 